\title{
A Method for Assessing Risk Rating of Natural Gas Pipeline Based on Accident Statistics
}

\author{
Xiaoyuan Xu, Xuanya Liu, Ge Guo, Chao Ji \\ Tianjin Fire Research Institute of MPS, No.110 Wei Jin Nan Road, Nankai District, \\ Tianjin, 300381 , China. E-mail: xuxiaoyuan@tfri.com.cn \\ Received 19 January 2012 \\ Accepted 12 March 2012
}

\begin{abstract}
This article collected 199 cases domestic natural gas pipeline leakage accidents, and used the mathematical methods and SPSS statistical analysis software to analysis these accident cases. Based on the analysis of accidents statistics and disaster reduction factors, the natural gas pipeline risk rating assessment method based on accident statistics was proposed according to Risk value method and Risk matrix analysis. Taking the gas pipelines in a certain city for example, the risk grade of the pipeline was analyzed by the method proposed in this article, and more rigorous and effective measures to reduce the pipeline risk were proposed.
\end{abstract}

Keywords: Accident statistics, Natural gas pipeline, Risk assessment, Risk matrix

\section{基于事故统计的天然气管道风险等级评估方法}

\author{
许晓元, 刘晅亚, 郭歌, 纪超 \\ 公安部天津消防研究所, 天津 300381
}

\begin{abstract}
摘要: 本文统计了 199 例国内天然气管道泄漏事故, 并利用数理统计方法及 SPSS 分析软 件对事故案例进行分析。在事故统计和降灾因素分析的基础上, 根据危险度取值以及风险 矩阵分析, 提出基于事故统计的天然气管道风险等级评估方法, 并针对某城市天然气管道, 利用本文提出的方法对其风险等级进行了评估, 并提出相应的改善措施以降低管道风险。
\end{abstract}

关键词: 事故统计, 天然气管道, 风险评估, 风险矩阵

\section{1. 引言}

近 20 年来, 随着天然气能源的开发和应 用, 国内的天然气管道事业蓬勃发展。据不完 全统计，截止 2004 年，国内已形成了四川、 西北、华北以及“西气东输”等天然气长输管道 网，管道总长已超过 $9000 \mathrm{~km}^{[1]}$ 。然而天然气 是易燃易爆危险性气体, 一旦天然气管道发生 泄漏, 如果处理不当, 则会发生爆炸、火灾等 大型灾害性事故, 对人类生命安全及财产造成 严重威胁。

目前国外对燃气管道的风险分析已开展 了很多研究, 天然气管道风险分析已经逐步规 范化。如1992年W.Kent.Muhlbauer详细阐述了 天然气管道评估模型和各种评估方法, 并在总 结美国前 20 年油气管道风险研究工作的基础 上提出了一种基于管道相对风险数的管道危 险度评价方法 ${ }^{[2,3]}$; 英国健康与安全委员会研 发了用于计算管道失效风险的MISHAP软件 包, 可用于评估某地区的个人风险及社会风
险; 而我国在油气管道风险分析方面的研究则 起步较晚, 潘家华 ${ }^{[4-6]}$ 利用专家评分法和相对 风险评估方法从腐蚀、设计、操作、泄漏等几 个方面对油气管道进行了风险分析; 陈利琼 ${ }^{[7]}$ 针对我国在役油气长输管道特点, 从风险分 析、风险判断和风险决策等方面研究了油气长 输管道的定量评价方法; 刘斐 ${ }^{[8,9]}$ 以火灾、爆 炸作为燃气管道泄漏后的主要事故场景, 对管 道发生泄漏的事故概率及其后果进行分析, 在此基础上进行个人风险和社会风险的定量 分析, 并基于个人风险的可接受水平制定人员 不受伤害的管道安全距离, 为管道的管理及规 划提供依据; 柳红卫 ${ }^{[10]}$ 在肯特法的基础上, 分 析了城市埋地天然气管道失效的可能性与失 效后果的影响因子, 并利用半定量风险矩阵来 分析天然气管道的风险等级; 刑志祥 ${ }^{[11]}$ 指出造 成事故灾害性后果的主要方式是热腐蚀和冲 击波, 并对天然气管道事故率、事故后果进行 了分析, 得出了热腐蚀引起人员死亡概率的计 
算方法; 翁永基 ${ }^{[12]}$ 利用事件树分析法对泄漏事 故的3种后果 (无燃烧、燃烧和爆炸事件) 发 生概率进行了估算，同时阐述了3种损伤模型

（速度、敏感、无关模型），对油气管道泄漏 事故定量风险评价方法进行了改进; 此外还有 其他学者对天然气管道的事故风险进行了研 究 ${ }^{[13-17]}$ 。

前人对管道的风险评价大多都基于理论 分析, 而理论结果取决于采用何种模型及边界 参数的设定, 其结果与实际有一定的差距。本 文统计了 199 例国内天然气管道泄漏事故, 并 利用数理统计方法及 SPSS 分析软件对事故案 例进行分析。在事故统计和降灾因素分析的基 础上, 根据危险度取值以及风险矩阵分析, 提 出基于事故统计的天然气管道风险等级评估 方法。由于本方法是基于国内天然气事故案例 的统计数据, 较前人的研究其针对性及准确性 有一定提高, 且使用起来较为便捷。

\section{2. 天然气事故数据统计及软件分析}

\section{1 天然气事故数据统计}

本文搜集了1919年至2011 年间国内天然 气泄漏、火灾爆炸事故案例 199 例, 并将这些 事故案例的各相关因素（时间、地点、事故原 因、管径、管道压力、壁厚、管道材料、管道 埋深、涂层、安装投产时间、事故类型、发生 此灾害的地点类型、经过、处置情况、经济损 失、人员伤亡事故等级) 进行了统计及分析。

表 1 为国内天然气泄漏事故原因统计。通 过表 1 可知, 国内天然气事故原因以第三方破 坏为主, 由此原因造成的事故占 $52.76 \%$, 第 三方破坏因素包括：施工外力破坏 $38.69 \%$ 、 重车碾压 $3.02 \%$ 、违规物占压管道 3.02\%、偷 盗 $1.51 \%$ 及人员故意破坏 $6.53 \%$; 自然因素造 成的损失占了 $11.06 \%$, 其中腐蚀占得比例最 大；设计不合理、施工不合理、设备缺陷、日 常维修不当、违规操作等因素分别占 $1.01 \%$ 、 $7.14 \% 、 3.02 \% 、 2.01 \% 、 3.52 \%$, 其中, 施工 不合理主要表现为焊接缺陷, 焊接缺陷表现 有: 埋弧焊缝裂纹, 电焊钢管沟状裂纹, 现场 焊缝未焊透、未熔合; 由于腐蚀、日常维护不 当等综合因素造成的事故占 $4.02 \%$ 。

国内天然气事故中，管道破损比例为 $59.88 \%$, 这主要是因为天然气事故大多是由施 工外力导致，施工时管道最易受到破坏; 破损 位置为焊缝的比例较高, 占 $33.14 \%$, 这是因 为国内较早的管道采用传统的手工焊方法, 这 种方法质量不易保证 ${ }^{[1]}$ 。

表 3 为天然气事故人为处置情况统计。处 置情况主要包括群众、消防队、天然气公司处 置三个方面。通过统计, 国内天然气事故中有 $75.97 \%$ 的事故处置较好, $15.5 \%$ 的事故处置较 差, $8.53 \%$ 的事故处置非常差。
国内天然气事故中只发生泄漏的事故占 $69.01 \%$; 发生火灾的事故占 $13.68 \%$, 发生爆 炸的事故占 $12.68 \%$; 发生了火灾及爆炸次生 灾害的事故占 $4.93 \%$ 。

表 1 国内天然气泄漏事故原因统计

\begin{tabular}{lcc}
\hline \multicolumn{1}{c}{ 事故原因 } & 事故次数 & 比例 $(\%)$ \\
\hline 第三方破坏 $\mathrm{A}$ & 105 & 52.76 \\
其中: 施工外力破坏 A1 & $(77)$ & $(38.69)$ \\
$\quad$ 重车碾压 A2 & $(6)$ & $(3.02)$ \\
$\quad$ 违规物占压管道 A3 & $(6)$ & $(3.02)$ \\
$\quad$ 偷盗 A4 & $(3)$ & $(1.51)$ \\
$\quad$ 人为故意破坏 A5 & $(13)$ & $(6.53)$ \\
自然因素 B & 22 & 11.06 \\
其中: 腐蚀 B1 & $(9)$ & $(4.52)$ \\
$\quad$ 自然条件 B2 & $(5)$ & $(2.51)$ \\
$\quad$ 地质条件变化 B3 & $(8)$ & $(4.02)$ \\
设计不合理 C & 2 & 1.01 \\
施工不合理 D & 14 & 7.14 \\
其中: 焊接缺陷 D1 & $(13)$ & $(6.63)$ \\
$\quad$ 开挖回填不良等 D2 & $(1)$ & $(0.50)$ \\
设备缺陷 E & 6 & 3.02 \\
日常维修、管理不到位 F & 4 & 2.01 \\
违规操作 G & 7 & 3.52 \\
其他 H & 7 & 3.52 \\
其中综合原因: A5 B2、B1 & 各 1 次 \\
F、D1 B1、 D2 B1、B3 C、 & & 4.02 \\
B3 F、C E、A5 F & & \\
合计 & 199 & 100 \\
\hline
\end{tabular}

\begin{tabular}{cccccc}
\multicolumn{7}{c}{ 表 2} & 国内天然气事故地区统计 \\
\hline 省份 & $\begin{array}{c}\text { 事故 } \\
\text { 数 }\end{array}$ & $\begin{array}{c}\text { 比例 } \\
(\%)\end{array}$ & 省份 & $\begin{array}{c}\text { 事故 } \\
\text { 数 }\end{array}$ & $\begin{array}{c}\text { 比例 } \\
(\%)\end{array}$ \\
\hline 四川省 & 26 & 16.7 & 广东省 & 3 & 1.94 \\
重庆市 & 20 & 12.9 & 辽宁省 & 2 & 1.29 \\
陕西省 & 14 & 9.03 & 黑龙江省 & 2 & 1.29 \\
北京市 & 9 & 5.81 & 新疆省 & 2 & 1.29 \\
江苏省 & 9 & 5.81 & 宁夏省 & 2 & 1.29 \\
安徽省 & 9 & 5.81 & 福建省 & 1 & 0.65 \\
上海市 & 8 & 5.16 & 甘肃省 & 1 & 0.65 \\
山东省 & 7 & 4.52 & 贵州省 & 1 & 0.65 \\
湖北省 & 6 & 3.87 & 天津市 & 1 & 0.65 \\
河南省 & 6 & 3.87 & 海南省 & 1 & 0.65 \\
吉林省 & 5 & 3.23 & 山西省 & 1 & 0.65 \\
湖南省 & 4 & 2.58 & 江西省 & 1 & 0.65 \\
河北省 & 4 & 2.58 & 云南省 & 1 & 0.65 \\
浙江省 & 4 & 2.58 & 广西省 & 1 & 0.65 \\
内蒙古 & 3 & 1.94 & & & \\
\hline
\end{tabular}

按照国家有关规定，事故按人员伤亡程度 和经济损失情况划分为小事故、一般事故、较 大事故、重大事故、特大事故、特别重大事故。 经过统计, 国内天然气事故中约 $80 \%$ 为一般事 故或小事故; 较大事故、重大事故、特大事故 分别为 $9.85 \% 、 7.04 \% 、 3.52 \%$; 特别重大事故 
占 $1.41 \%$ 。

表 3 处置是否得当统计

\begin{tabular}{ccc}
\hline 处置情况 & $\begin{array}{c}\text { 事故 } \\
\text { 数 }\end{array}$ & $\begin{array}{c} \\
(\%)\end{array}$ \\
\hline
\end{tabular}

及时发现泄漏并报警，消防、

天然气公司人员及时到位并处置 $98 \quad 75.97$

得当、及时疏散人群

短时间内未及时发现泄漏或 报警较晚或短时间内未及时疏散 人群并且消防及天然气公司人员 $20 \quad 15.50$ 处置较得当

长时间未发现泄漏或未疏散 人群或消防、天然气公司处置不当 $11 \quad 8.53$

\section{2 软件分析}

SPSS 是专业的通用统计软件, 它是一个 组合式软件包, 兼有数据管理、统计分析、统 计绘图和统计报表功能。相关分析是用于描述 两个变量间联系的密切程度。

本文利用 SPSS 统计分析软件, 将事故案 例中的事故原因、破裂位置、事故类型、处置 情况等因素与事故案例后果之间做了相关性 分析, 分析结果见表 4。表 4 中, 数据为各影 响因素与事故后果之间相关性的绝对值, 其数 值越大说明该因素与事故后果之间相关性越 强, 即处置情况与事故后果的相关性最高, 事 故类型其次, 破裂位置与事故后果的相关性最 差。

表 4 因素与后果之间的相关性

\begin{tabular}{cc}
\hline 影响因素 & 与事故后果相关性的绝对值 \\
\hline 处置情况 & 0.527 \\
事故类型 & 0.353 \\
事故原因 & 0.189 \\
破裂位置 & 0.089
\end{tabular}

3. 基于事故统计的天然气管道风险等级评 估方法

表 5 基于事故统计的天然气管道风险等级评估表

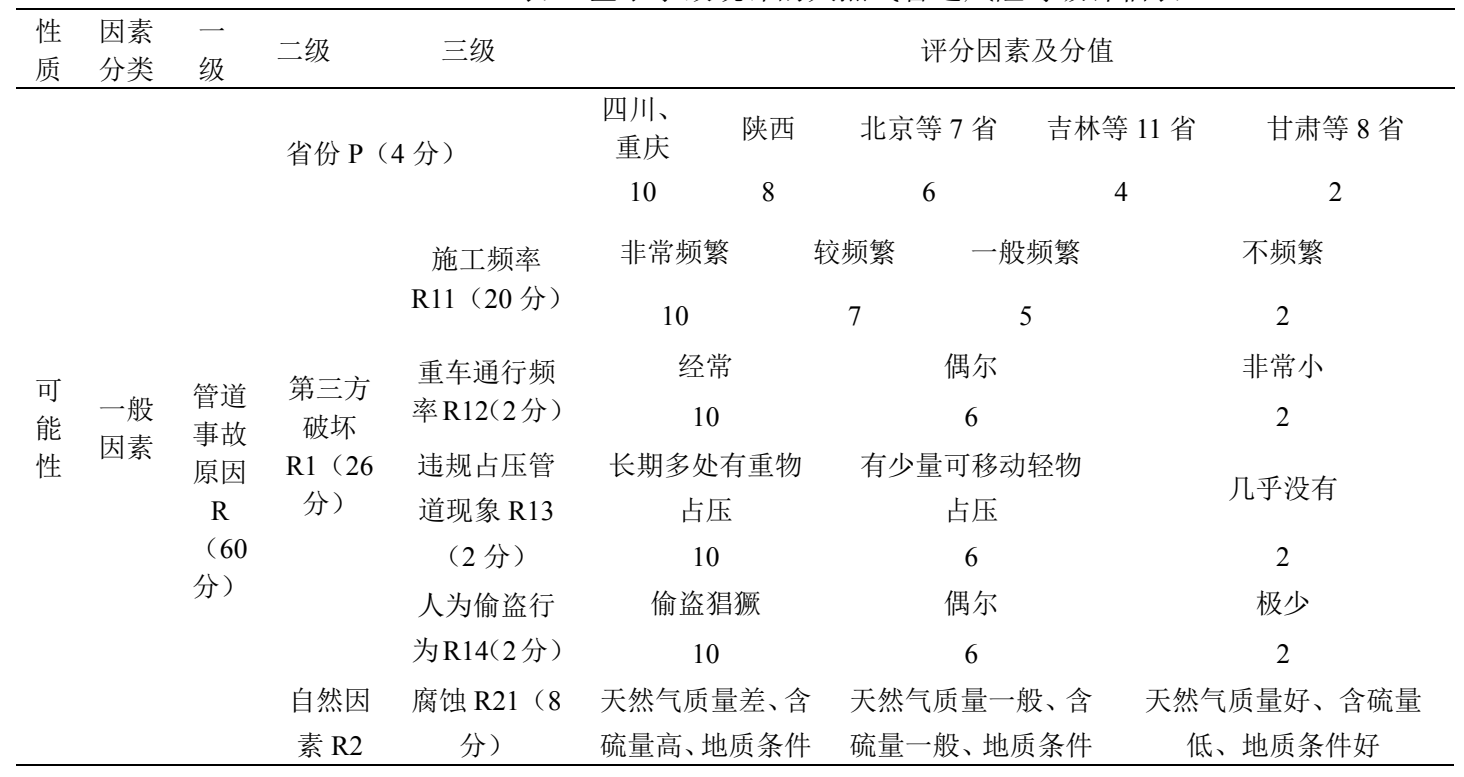

Published by Atlantis Press

Copyright: the authors

63

\section{1 评估方法的建立}

通过对事故的分析可知，城市燃气事故后 果与救灾系统及预警系统的完善度、抗灾能力 的大小、应急能力等降灾因素有着密切的关 系, 所以本评估方法将各因素划分为一般因素 及降灾因素两大类。

通过对 199 例国内事故分析可知: 天然气 管道事故发生的可能性与管道所在省份、管道 遭遇第三方破坏可能性、管道周围的自然因 素、管道设计、管道施工、设备缺陷、天然气 公司工作人员违规操作的可能性、管道的壁 厚、埋深等一般因素有关, 还与相关降低管道 发生泄漏的技术及管理措施等降灾因素有关。 这些技术和措施包括: 天然气泄漏预测预警技 术、天然气公司对管道的日常维护、政府及群 众对天然气管道的维护等等。而天然气发生事 故的严重性则与管道周围人员建筑密集程度、 管道直径、管道压力等一般因素有关, 还与群 众、天然气公司、消防对天然气事故的处置等 降灾因素有关。基于事故统计的天然气管道风 险等级评估表见表 5, 通过赋值的方法计算管 道发生事故的可能性及事故严重性的分值, 并 通过表 6 (失效可能性等级划分标准)、表 7 (失效严重性等级划分标准) 来确定管道失效 可能性及事故严重性的等级。

事故风险是事故可能性与事故后果严重 性的乘积。将天然气管道失效可能性与管道事 故后果严重性分别作为横纵坐标绘制成风险 矩阵表，见表 8 。通过此表可以判断管道风险 的大小, 如表 8 中网格区域为高风险区域, 要 及时采取措施以降低管道的现有风险, 竖纹区 的可能性很大要么发生事故后非常严重, 也应 找出原因积极采取有效措施, 斜纹区域为可接 受的低风险区域。 域为中等风险区域, 此种情况时要么发生事故 


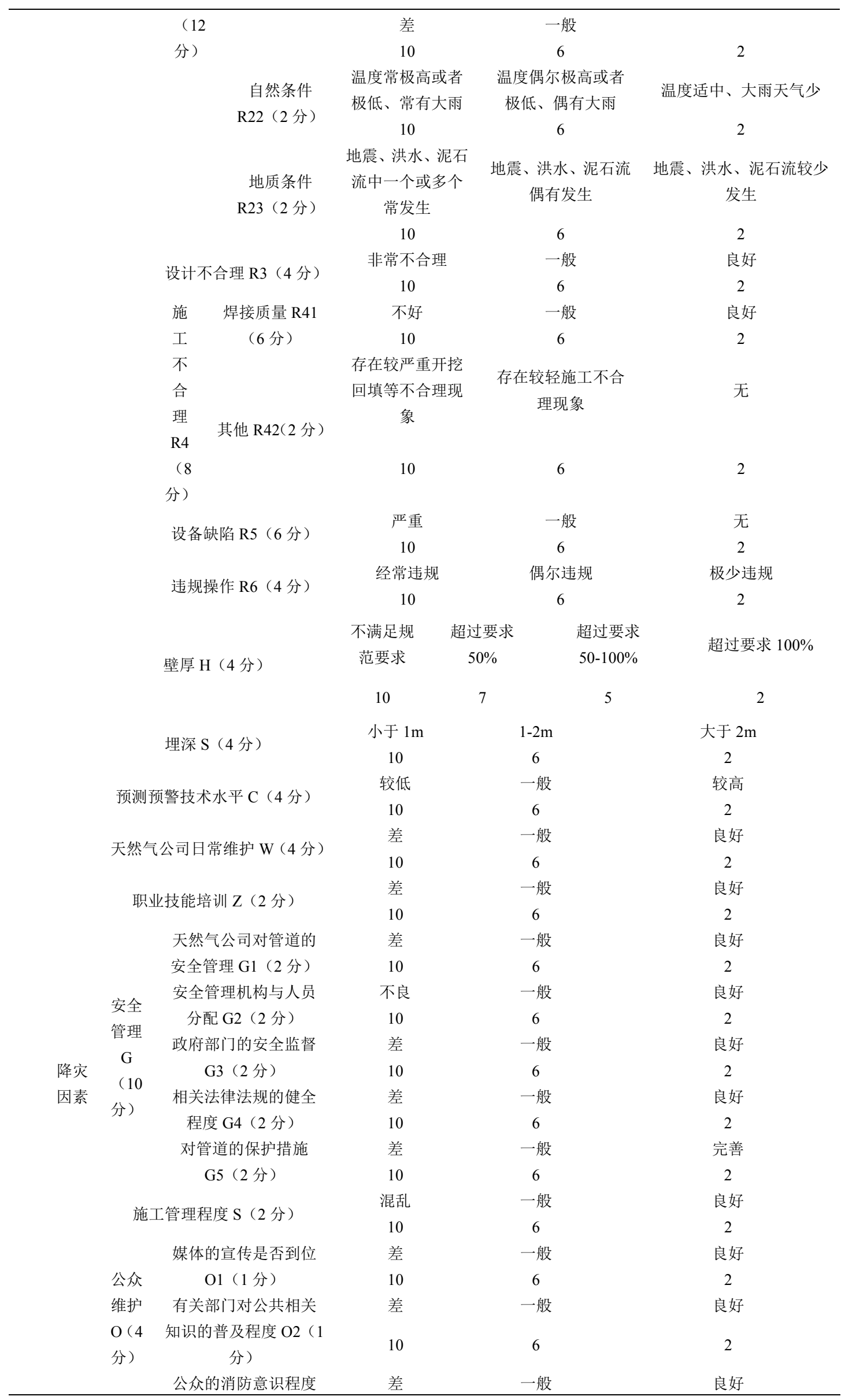




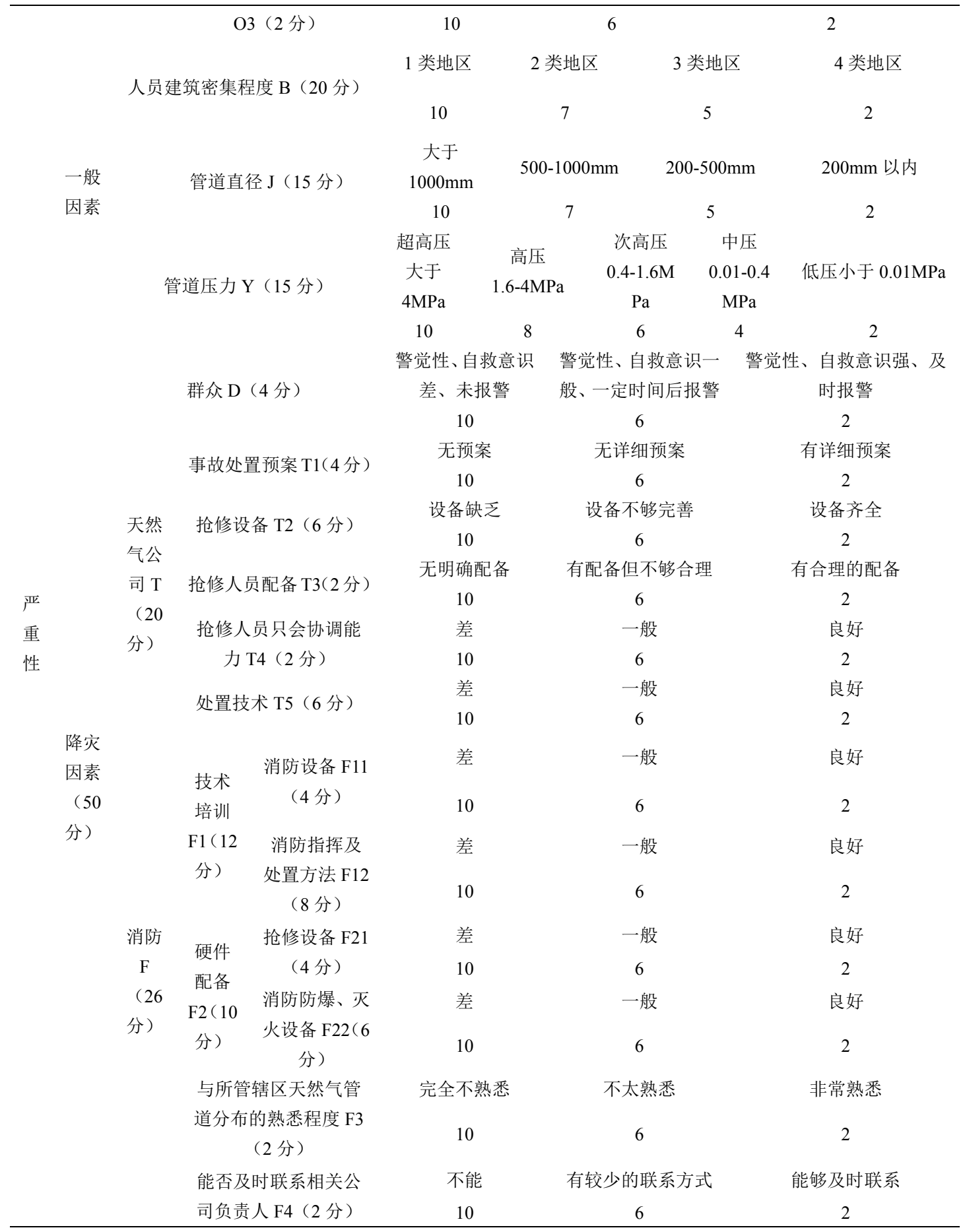

表 6 失效可能性等级划分标准

\begin{tabular}{lc}
\hline 分值 & 失效可能性等级级别 \\
$(0,25]$ & 可能性 \\
$(25,50]$ & 几乎不可能 \\
$(50,75]$ & 可能性较小 \\
$(75,100]$ & 可能性较大 \\
\hline
\end{tabular}

表 7 失效严重性等级划分标准

\begin{tabular}{ccc}
\hline 分值 & $\begin{array}{c}\text { 失效严重性等级 } \\
\text { 级别 }\end{array}$ & 严重性 \\
\hline$(0,25]$ & 1 & 不严重 \\
$(25,50]$ & 2 & 不太严重 \\
$(50,75]$ & 3 & 较为严重 \\
$(75,100]$ & 4 & 非常严重 \\
\hline
\end{tabular}


表 8 天然气事故风险矩阵表

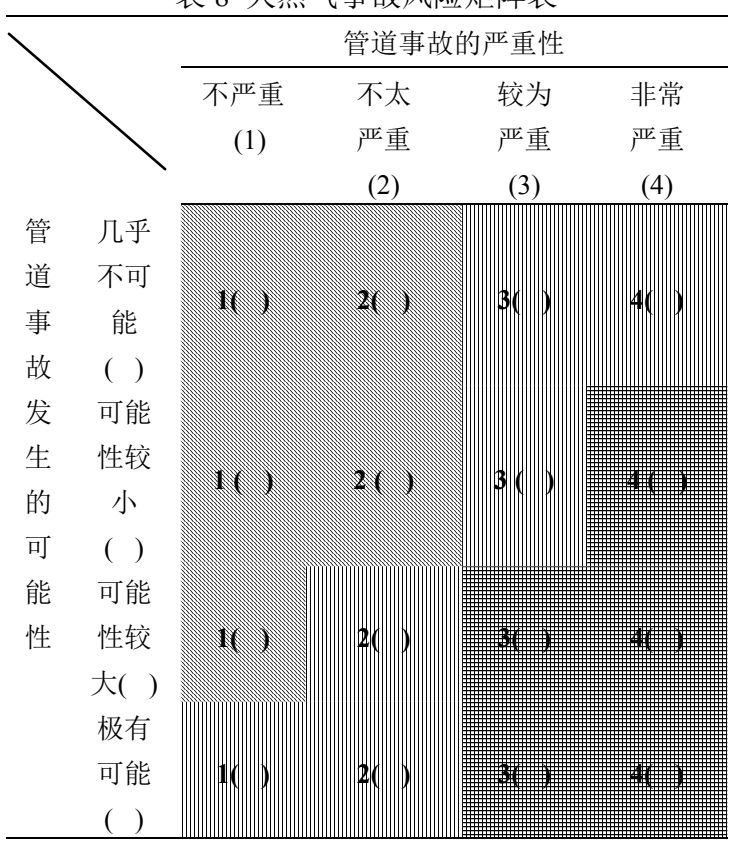

\section{2 评估方法中因子及数值的确定}

表 5 基于事故统计的天然气管道风险等 级评估表中因子及数值的确定方法如下:

通过对已搜集的 200 余例天然气事故进 行统计分析, 根据各事故影响因素对事故影响 的重要度来确定风险体系表中一级、二级及三 级影响因素的取值。根据实际情况, 影响因素 可以增加, 新增加因素分值也可以通过专家打 分的方式得到。

通过表 2 可知, 四川省、重庆市事故比例 最高, 陕西次之, 甘肃等省份所占事故比例最 低, 所以, 将天然气管道所处省份划分为 5 个 等级, 最容易发生事故的省份赋值为 10 分, 最不易发生事故的省份赋值为 2 分。根据表 1 可知, 天然气管道天然气管道容易遭受第三方 破坏, 而天然气管道所在位置的施工频率、重 车通行频率、违规占压管道现象、人员偷盗猖 獗程度均是第三方破坏的重要因素, 对各因素 发生可能性进行分级并赋予分值, 最高为 10 分; 同理对天然气管道发生泄漏事故的可能性 各相关因素进行分级并赋值，见表 5。

天然气事故附近的人口、建筑密集程度是 事故后果严重性的一个重要因素, 按照《输气 管道工程设计规范GB 50251-2003》, 将管道 所经之处分为 4 类地区, 1 类地区人口最少, 4 类地区人口最多, 见表 9 。

管道的直径及管道压力的大小也与事故 后果严重性有一定关系, 将管道直径划分为 4 个等级并赋予相应的分值, 管道压力按《城镇 燃气设计规范 GB50028-2006》划分为 5 个等 级。如表 4 中所示, 群众、天然气公司、消防 队对天然气事故的处置情况是事故后果的重 要影响因素, 根据群众消防意识、天然气公司
的应急能力及消防队业务能力分为相应的等 级并赋值。

\begin{tabular}{cc}
\multicolumn{2}{c}{ 表9 人口、建筑密集程度分类标准 } \\
\hline 由地区类别 & $\begin{array}{c}\text { 由管道中心线向两侧各 } 200 \mathrm{~m} 、 \text { 沿 } \\
\text { 管道长 } 2000 \mathrm{~m} \text { 的长方形区域内的 } \\
\text { 人口状况 }\end{array}$ \\
\hline 1类地区 & 少于等于 15 户 \\
2 类地区 & 多于 15 户少于 $100 户 ~$ \\
3类地区 & 等于多于 $100 户 ~$ \\
4类地区 & 有4层及4层以上楼房(不计地下室 \\
& 层数) 集中、交通频繁、地下设施 \\
& 多的地段 \\
\hline
\end{tabular}

注: 1. 在规定面积内虽然住户少于100户, 但有学校、 医院以及其他公共场所等人群聚集的地方 也应归为 3 类地区。

2. 当划分地区等级边界线时, 边界线距最近 一栋建筑物外边缘大于或等于 $200 \mathrm{~m}$ 。

3. 当一个地区的发展规划, 足以改变地区的 现有等级时，应按发展规划划分地区等 级。

\section{4. 基于事故统计的天然气管道风险等级评 估方法的应用}

以某段天然气管道为例。天然气公司或者 政府部门可以应用本文建立的基于事故统计 的天然气管道风险等级评估方法来对此段天 然气管道的风险等级进行分析。经调研, 此段 天然气管道相关因素如下: 该天然气管道位于 四川省; 该天然气管道直径 $325 \mathrm{~mm}$, 壁厚 $6.4 \mathrm{~mm}$, 埋深 $1.2 \mathrm{~m}$, 管道压力为中压; 该管道 位于某市区中心区域, 施工频率较为频繁, 该 市区的施工管理制度较为严格, 极少有重车通 过，未发现有违规占压管道现象，该地区极少 出现偷盗天然气现象; 由于天然气直接供附近 居民使用, 天然气质量较高, 含硫量少, 但该 地区土壤成酸性; 该地区经常会有大到暴雨天 气，偶尔有小型地震发生; 经调查, 该管道设 计较为合理, 但由于技术问题焊缝存在一定缺 陷, 暂未发现有缺陷设备, 且预测预警技术不 成熟; 该地区人员素质较高, 消防意识较强; 但天然气公司管理松弛, 日常维护不到位, 按 时进行职业技能培训, 但质量不高, 应急预案 不够详细明确, 偶尔会发生员工违规操作现 象; 政府部门及天然气公司对管道保护措施较 全面, 但政府部门未明确设置监管部门; 该范 围内的消防队业务过关, 设备精良, 指导员在 处置天然气事故方面有较丰富的经验, 熟悉所 管辖区天然气管道分布及相关负责人的联系 方式。利用本文提出的基于统计的天然气管道 风险等级评估方法建立表 10 。 
表 10 四川某段天然气管道风险评估表赋值



经计算，并与表6、7进行比对可知，该天 然气管道发生事故的可能性为 级, 一旦发生 事故, 其后果严重程度为 3 级, 由表 8 可知, 该 天然气管道风险等级为高等。由于该管道风险 等级较高, 该地区天然气公司及政府要及时采 取措施以降低管道的现有风险, 如: 降低施工 频率、加强施工管理制度、天然气公司应加强 天然气管道的日常维护、对员工应加强培训以
杜绝违规操作现象、制定详细的应急预案等, 以更大程度上保障居民及财产安全。

\section{5. 总结与展望}

本文通过对统计的事故进行分析, 在事故 分析结果的基础上并考虑降灾因素利用危险 度取值法及风险矩阵的思想提炼出基于事故 统计的天然气管道风险等级评估方法。此外, 
本文结合具体的工程应用分析, 利用本文提出 的基于事故统计的天然气管道风险等级评估 方法对某段管道进行了评估, 并提出相应的改 善措施以降低管道风险。

本文提出的基于事故统计的天然气管道 风险等级评估方法中各因素的权重值基于统 计数据, 有理有据, 符合中国国情, 应用便捷, 结果清晰, 可尽快对存在风险的管道提出相应 的改善措施。对于具体的管道可增加相关评估 的因素以得到更为准确全面的结果。

\section{参考文献}

1. Zheng Jinyang, Ma Xiakang, Yin Xieping. Safety risk identification, assessment and control of Long-distance pipeline(Chemical Industry Press, Beijing,2004).

郑津洋, 马夏康, 尹谢平.长输管道安全风险辨 识 评价 控制[M].北京: 化学工业出版社, 2004

2. Muhlbauer, Pipeline Rsik Magement Manual 1st edn. (Gulf Publishing ComPany, Houston, Texas, 1992)

3. W.Kent Muhlauer, Pipeline Rsik Magement Manual 2nd edn(Gulf Publishing ComPany, Houston, Texas, 1996)

4. Pan Jiahua. Risk analysis on oil and gas pipeline (1),J. Oil \& Gas Storage and Transportation. 14(3)(1995)11-15

潘家华, 油气管道的风险分析(待续) [J].油气储 运, 14(3), 1995:11-15

5. Pan Jiahua. Risk analysis on oil and gas pipeline (2),J. Oil \& Gas Storage and Transportation. 14(4)(1995)1-7

潘家华, 油气管道的风险分析(续一) [J].油气储 运, 14(4), 1995:1-7

6. Pan Jiahua. Risk analysis on oil and gas pipeline

(2),J. Oil \& Gas Storage and Transportation. 14(5)(1995)3-10

潘家华, 油气管道的风险分析(续完) $[\mathrm{J}]$.油气储 运, 14(5), 1995:3-10

7. Chen Liqiong.Supported by Middle-Young People Innovation Foundation of Petroleum Science and Technology, PhD thesis, Chengdu: Southwest Petroleum University, (2004).

陈利琼. 在役油气长输管线定量风险技术研究 (D). 博士论文.西南石油学院 2004

8. Liu Fei, Liu Mao, Quantitative Risk Analysis for Urban Pipeline Carrying Natural Gas, J. Acta Scientiarum Universitatis Nankaiensis. 39(6)(2006)31-35

刘斐,刘茂.城市燃气管线的定量风险分析 [J].南 开大学学报(自然科学版),2006,39(6):31-35

9. Liu Fei, Liu Mao, Quantitative Risk Analysis for Fire of Urban Transmission Pipeline Release, $J$. Safty and Environmental Engineering. 13(4)
(2006)63-65

刘斐,刘茂.城市输气管线火灾事故的风险定量

计算[J].安全与环境工程,2006,13(4):63-65

10. Liu Hongwei, Study on semi-quantitative risk assessment method for urban natural gas pipeline, J. Journal of Safety Science and Technology. 2(3) (2006)96-100

柳红卫.城市天然气管道半定量风险评估方法研 究 [J].中国安全生产科学技术, 2006,2(3) :96-100

11. Xing Zhixiang, A quantitative method of risk assessment of natural gas pipeline, J. China Petroleum Machinery. 36(4) (2008)15-17

刑志祥.天然气长输管道的定量风险评价方法 [J]. 石油机械.2008,36(4):15-17

12. Weng Yongji, Quantitative risk assessment for failure of oil and gas pipelines, J. Acta Petrolei Sinica. 25(5) (2004)108-112

翁永基.油气管道泄漏事故的定量风险评价 $[\mathrm{J}]$. 石油学报.2004,25(5) :108-112

13. Xu Yabo, Qian Xinming, Liu Zhenyi, Quantitative Risk Analysis on the Leakage of Compressed Natural Gas Pipeline, J. China Safety Science Journal. 18(1) (2008)146-149 徐亚博,钱新明,刘振翼.天然气输送管道泄漏事 故危害定量分析 [J]. 中国安全科学学 报,2008,18(1):146-149

14. Cao Zheng, Liu Mao, Individual Risk Analysis and Researches of City Gas Pipelines, J. Risk Management. 4(17) (2009)21-25 操铮, 刘茂.城市燃气管线的个人风险分析与研 究[J].风险管理,2009,4(17)21-25

15. Gao Junbo, Guo Yue, Wang Xiaofeng, Research of Quantitative Risk Analysis Model for Urban Gas Pipeline Network, J. Journal of Basic Science and Engineering. 16(12) (2008)280-286

高俊波,郭越,王晓峰.城市燃气管网的定量风险 分析模型研究 [J]. 应用基础与工程科学学 报,2008,16(12):280-286

16. Guo Zhanglin, Sun Yuli, Safety asseccment of urban gas pipelines based on the risk analysis, J. Journal of Hebei University of Engineering(Natural Science Edition) . 27(2) (2010)87-90

郭章林,孙雨丽.基于风险分析的城市燃气管道 安全评价 [J]. 河北工程大学学报 (自然科学 版).2010，27(2):87-90

17. Yang Linjuan, Shen Shiming, Quantitative risk analysis of natural gas distribution pipeline network, J. Pressure vessel . 24(9) (2007)29-34 杨林娟, 沈士明.天然气配气管网定量风险分析 [J].压力容器,2007,24(9):29-34 